\title{
ESTIMATION OF LOCAL LIQUID FILM THICKNESS IN TWO- PHASE ANNULAR FLOW
}

\author{
BO AN LEE ${ }^{1}$, BYONG JO YUN ${ }^{2}$, KYUNG YOUN KIM ${ }^{3}$, SIN KIM ${ }^{1,4^{*}}$ \\ ${ }^{1}$ Department of Nuclear and Energy Engineering, Jeju National University \\ Jeju 690-756, Korea \\ ${ }^{2}$ Department of Mechanical Engineering, Pusan National University \\ Busan 609-735, Korea \\ ${ }^{3}$ Department of Electronic Engineering, Jeju National University \\ 690-756, Korea \\ ${ }^{4}$ Institute for Nuclear Science and Technology, Jeju National University \\ Jeju 690-756, Korea \\ *Corresponding author. E-mail : sinkim@jejunu.ac.kr
}

Received February 28, 2011

Accepted for Publication June 20, 2011

In many semi-empirical analyses of flow boiling heat transfer, an annular flow is often assumed as a model flow and the local liquid film thickness is a key parameter in the analysis. This work considers a simple electrical conductance technique to estimate the local liquid film thickness in two-phase annular flows. In this approach, many electrodes are mounted flush with the inner wall of the pipe. Voltage differences between two neighboring electrodes for concentric annular flows with various liquid film thicknesses are obtained before the main experiments and logged in a look-up table. For an actual application in the annual flow, voltage differences of neighboring electrodes are measured and then corresponding local film thicknesses are determined by the interpolation of the look-up table. Even though the proposed technique is quite simple and straightforward, the numerical and static phantom experiments support its usefulness.

KEYWORDS : Liquid Film Thickness, Annular Flow, Electrical Conductance

\section{INTRODUCTION}

Two-phase heat transfer is one of the important heat transfer mechanisms in many types of heat exchangers due to its heat transfer characteristics, which are superior to those of a single phase flow. Hence, it has drawn interest from a number of researchers for a long time. In many semi-empirical analyses for flow boiling heat transfer, slug flows with elongated bubbles or annular flows are often assumed as reference flow patterns and in the analysis the liquid film thickness is treated as one of the main parameters $[1,2]$. Once the local liquid film thickness is determined, it becomes easier to estimate the two-phase parameters, such as the void fraction and the liquid and gas velocities, as well as the acceleration pressure drop and the fluid inventory along the channels [3].

Measuring the liquid film thickness in a two-phase annular flow has been an importance issue for a long time and many methods have been developed for it. The

\footnotetext{
${ }^{*}$ Corresponding author: sinkim@jejunu.ac.kr
}

methods can be classified into four groups according to their measurement principles: 1) optical, 2) acoustic, 3) radiological and 4) electrical methods. In optical methods, various optical devices, such as high speed cameras and lasers, are used for detecting an interface $[4,5]$, total internal light reflection [6], fluorescence intensity [7] and so on. Acoustic methods are based on the fact that ultrasonic waves are reflected at the gas-liquid interface and the time interval between the emitted and the reflected signal is converted into the film thickness [8,9]. Radiological approaches use radiation, such as X-ray, gamma-ray, and neutrons. In radiological methods, the different attenuation characteristics in gas and liquid are used to measure the liquid film thickness $[10,11]$. Electrical methods are classified into two approaches: conductance and capacitance. The conductance (or the capacitance) between electrodes placed on the inner surface of the pipe is dependent on the conductivity (or the dielectric constant) values of each phase and its morphology. The conductance method is cheaper than the other methods: It is non-intrusive and does not disturb flows. In addition, the response to the change of the liquid film thickness is very fast. Coney 
[12] proposed a conductance probe composed of two parallel electrodes with unequal widths and separated by an insulator to measure liquid film thickness and developed a theoretical model to analyze the relationship between the conductance and the liquid film thickness. Fukano [13] suggested in-line and concentric ring-type probes and successfully applied them to the liquid film thickness measurement in a rectangular test section. Conte et al. [14] and Geraci et al. [15] proposed flush-mounted pin probes. The method is used for very thin films, typically up to 2 $\mathrm{mm}$ (pipe diameter $=127 \mathrm{~mm}$ ). However, that work dealt with measuring thicker film up to $11 \mathrm{~mm}$ (pipe diameter $=80 \mathrm{~mm}$ ). More recently, Liu et al. [16] used Electrical Capacitance Tomography (ECT)-based image reconstruction to extract the liquid film thickness information.

The present work considers a conductance method to estimate the local liquid film thickness in an annular twophase flow through a circular pipe. Many electrodes are mounted flush with the inner wall of the pipe. Neighboring electrodes are selected as current carrying electrodes in turn. Voltage differences between the neighboring electrodes for concentric annular flows with various liquid film thicknesses are obtained before actual experiments and logged in a look-up table. For an annular flow, voltage differences between neighboring current carrying electrodes are measured and then corresponding film thicknesses are determined by the interpolation based on the look-up table. To show the performance, extensive numerical and static phantom experiments were conducted. As reviewed above, the idea of applying a conductance sensor to the measurement of the liquid film thickness is not new. However, to the authors' best knowledge, the attempt to estimate the peripheral distribution of the liquid film thickness in an annular flow through a circular pipe has not yet been reported. In fact, this measurement set-up is the same one used conventionally for Electrical Resistance Tomography (ERT), in which the resistivity distribution is reconstructed on the basis of the relationship between the injected currents and the induced voltages, and for the reconstruction the so-called inverse algorithm should be used [17].

This work will show numerically and experimentally that the liquid film thickness or the interface in annular flows can be estimated quite successfully without any inverse algorithm to reconstruct the resistivity distribution or to estimate the interfacial boundary while using an ERT measurement set-up.

\section{PROCEDURE FOR ESTIMATION OF LIQUID FILM THICKNESS}

This work considers an annular flow composed of liquid background and gas core as shown in Fig. 1. The resistivity values for the liquid and gas phases are $\rho_{l}$ and $\rho_{g}$, respectively. Here, $\rho_{l}$ is assumed to be constant and $\rho_{g}$ is set to infinity. A total of $L$ electrodes $\left(e_{1}, e_{2}, \cdots, e_{\mathrm{L}}\right)$ mounted flush with the inner wall of the pipe with radius $r$ are evenly positioned. The width and height of the electrode are $2 \Delta$ and $H$, respectively. The present work considers two types of electrical current injection patterns. In current pattern 1, pairs consisting of two adjacent electrodes are selected for the current carrying electrodes in order. That is, $L$ pairs of electrodes $\left(e_{1}, e_{2}\right),\left(e_{2}, e_{3}\right), \ldots$, $\left(e_{L}, e_{1}\right)$ are excited in turn and then the voltage differences of the corresponding electrode pairs are measured. This is the conventional adjacent current injection protocol in ERT. In current pattern $2, L$ pairs consisting of every other electrode $\left(e_{1}, e_{3}\right),\left(e_{2}, e_{4}\right), \ldots,\left(e_{L}, e_{2}\right)$ are chosen. For each current pattern, the normalized voltages difference $\Delta V^{*}$, defined by Eq. (1) with respect to the normalized liquid film thickness $\delta^{*}$ defined by Eq. (2), are plotted as in Fig. 2:

$$
\begin{gathered}
\Delta V^{*}=\frac{\Delta V-\Delta V_{w}}{\Delta V_{w}} \\
\delta^{*}=\frac{\delta}{r}
\end{gathered}
$$

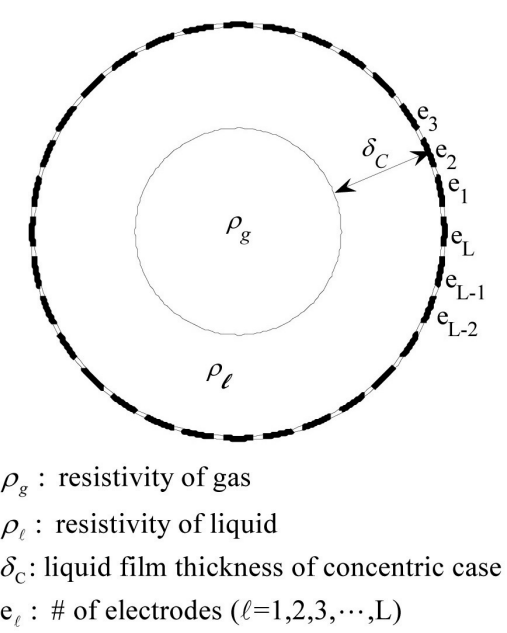

Fig. 1. Problem Domain Description for Concentric Annular Flow.

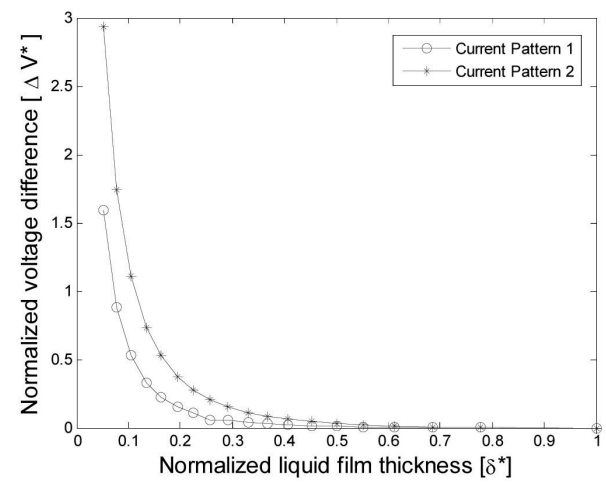

Fig. 2. Normalized Voltage Difference with Respect to Normalized Liquid Film Thickness. 
where $\Delta V$ is the voltage difference for the given liquid film thickness $\delta$ and $\Delta V_{w}$ denotes the voltage difference for $\delta$ $=r$, which corresponds to zero void fraction. Once the look-up table that relates $\Delta V^{*}$ to $\delta^{*}$ for the concentric annular flow is prepared from a calibration test, then the liquid film thickness can be determined from the look-up table using the measured voltage for a given annular flow. In general, conductivity is a function of the temperature and the voltage difference is inversely proportional to the conductivity. Once the temperature is known, the conductivity can be found from the material handbooks and the voltage difference for the reference temperature used for the generation of the look-up table can be calculated by multiplying the ratio of the conductivity at the present temperature to that at the reference temperature.

The procedure for estimating the liquid film thickness is summarized as follows:

(i) Create the look-up table of the normalized voltage differences between the preset electrode pairs for various liquid film thicknesses in a concentric annular flow;

(ii) the voltage differences for the electrode pairs for an arbitrary annular flow;

(iii) Estimate the liquid film thickness by consulting the look-up table.

To verify the present method, we conducted numerical experiments using COMSOL, which is a finite element method-based multi-physics software. In the simulation, this work treated the pipe as a unit circle and there were $L=32$ electrodes with a width of $2 \Delta=0.15$. The first numerical experiment (Case 1) involved various annular flow patterns with non-circular gas cores for void fractions in the range of $0.63-0.78$ as seen in Fig. 3. To evaluate
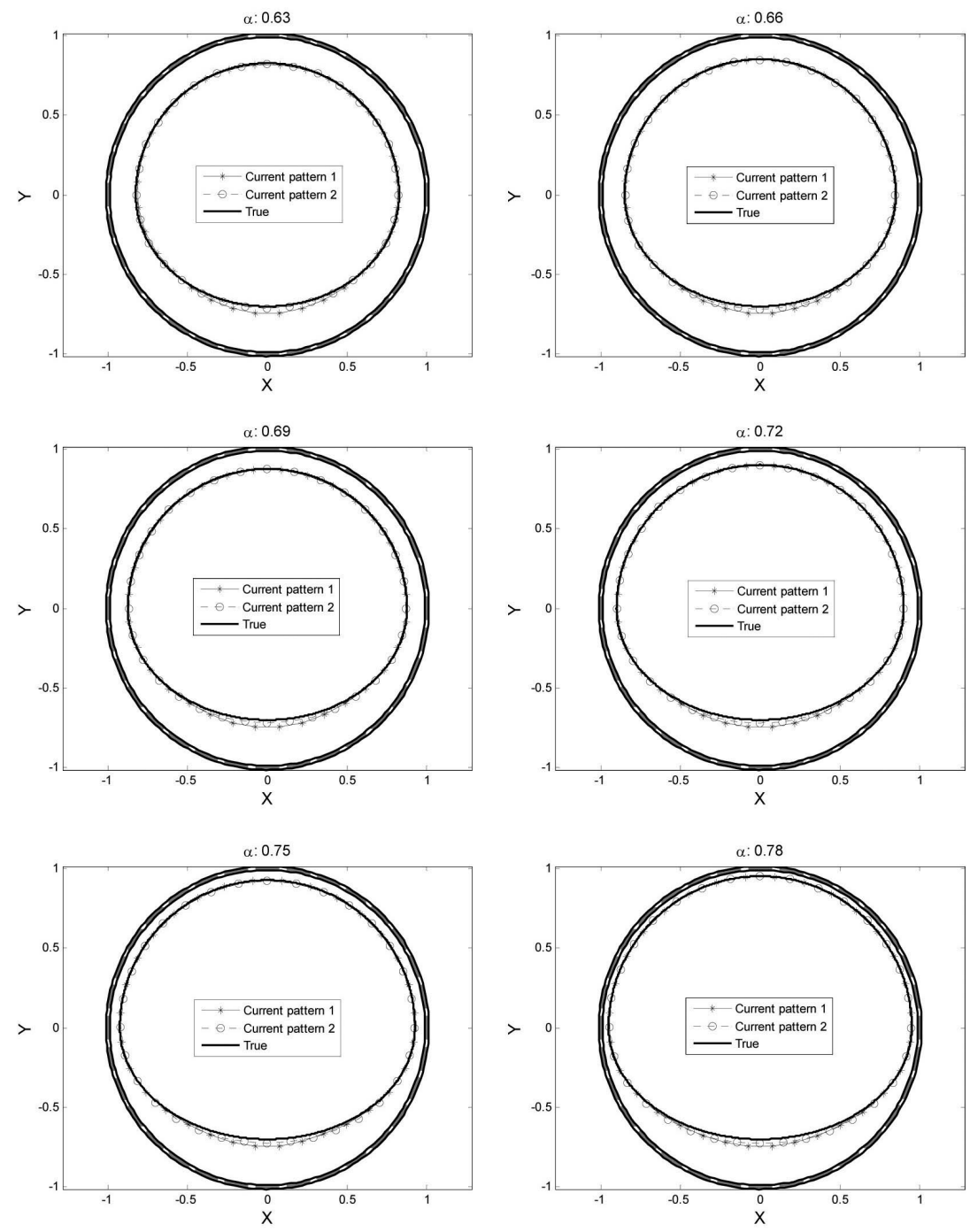

Fig. 3. Estimated Interface for Case 1. 
the estimation performance quantitatively, the estimation error was defined as

$$
E R R=\sqrt{\frac{1}{n} \sum_{i=1}^{n}\left[\frac{\delta_{\text {true }}(i)}{r}-\frac{\delta_{\text {estimated }}(i)}{r}\right]^{2}} .
$$

The estimation error was less than $0.18 \%$ as illustrated in Fig. 4.

In the second numerical experiment (Case 2), the void fraction was fixed while the gas core was moved from the center to the wall of the channel to investigate the effect of the gas core location on the estimation performance. For a void fraction of 0.72 , the gas core was moved toward the electrode and toward the gap between neighboring electrodes, respectively, as depicted in Fig. 5. The estimation errors were less than $2.5 \%$ as shown in Fig. 6 . The larger error of Case 2 may have been due to the fact that the liquid film in the other side becomes thicker as the gas core moves toward the pipe wall.

The last numerical experiment (Case 3) considered six distorted gas cores. The estimated results and the

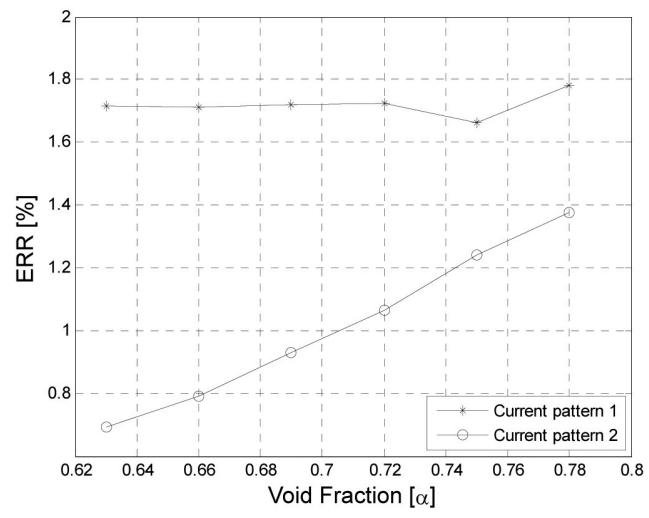

Fig. 4. Estimation Error for Case 1.

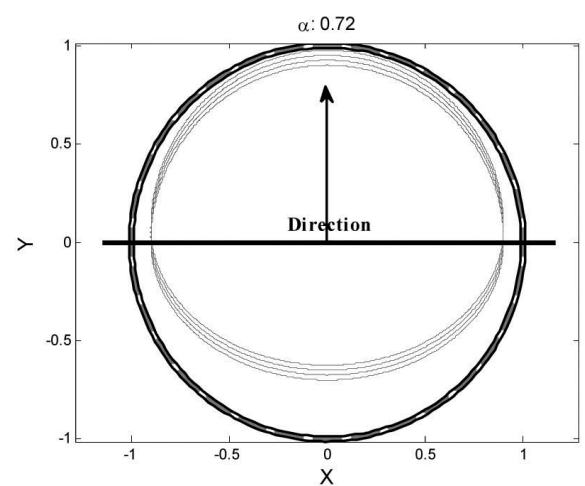

(a) Scenario I estimation error are shown in Figs. 7 and 8, respectively. The maximum error was less than $0.5 \%$.

For more extensive verification of the estimation performance, static phantom experiments were also conducted. A circular acrylic pipe with a radius of $40 \mathrm{~mm}$

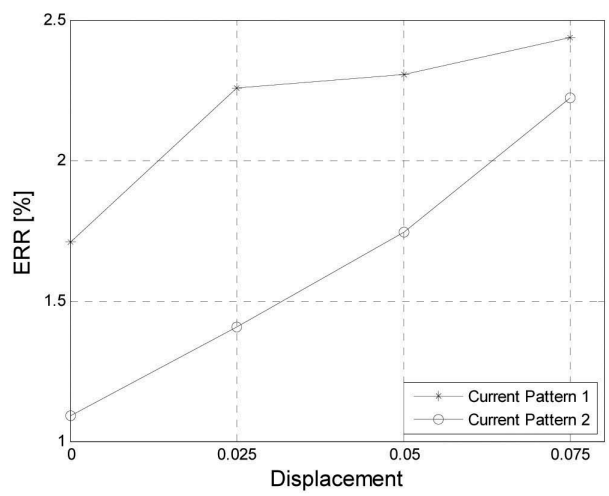

(a) Scenario I

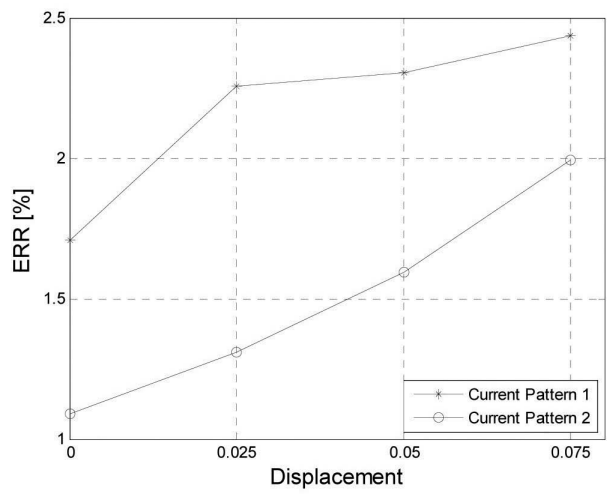

(b) Scenario II

Fig. 6. Estimation Error for Case 2.

Fig. 5.Gas Core Movement Scenarios for Case 2. 
was used. It was equipped with 32 flush-mounted electrodes with a width of $6 \mathrm{~mm}$ and a height of $20 \mathrm{~mm}$. Ten 200 $\mathrm{mm}$ high cylindrical acrylic rods simulating concentric gas cores were fabricated to obtain the void fractions of $0.1,0.2, \ldots, 0.9$, and 0.95 by changing the radius. The true void fractions were measured as $0.099,0.200,0.301$, $0.405,0.499,0.599,0.699,0.808,0.900$, and 0.948 in the static phantom experiments. It should be noted that in real experiments the voltage differences between electrode pairs will be different because of non-uniform electrode conditions, including imperfect positioning and different surface conditions even though the geometries are concentric annuli. The irregularity of the electrodes based on the voltage difference between adjacent electrodes for a pipe filled with water only is about $1.6 \%$. Hence, each electrode pair should have its own look-up table according to each void fraction.

In the present study, an Agilent 4284A LCR meter was used as a current source. The digital multimeter (NI PXI4065) with a switch (NI PXI-2536) was used to measure voltages. The measurement error for the voltage was estimated as less than $1.0 \%$ of the full scale. A current of $10 \mathrm{~mA}$ was applied to the selected electrodes and then the voltage difference was measured on the same electrodes. To simulate various patterns of the annular flow with a 0.69 void fraction, a non-circular acrylic rod was fabricated. In Fig. 9, the estimated interfaces for eight locations were successfully compared with the true ones. The first five

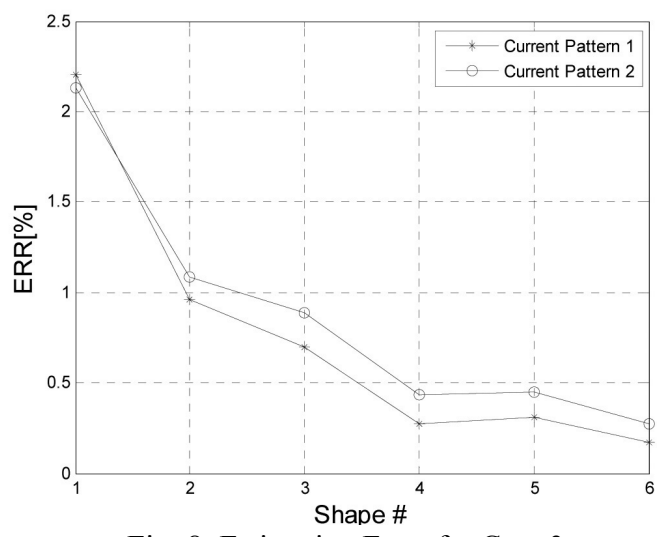

Fig. 8. Estimation Error for Case 3.

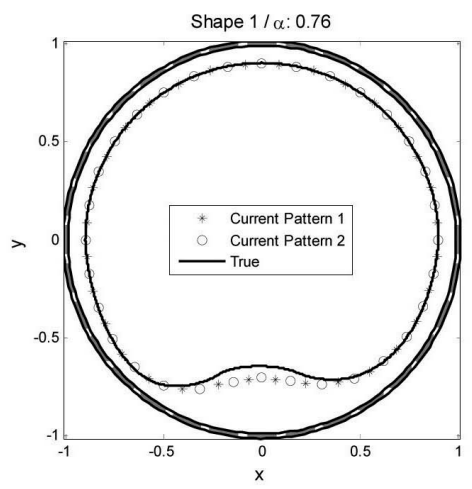

(a) Shape 1

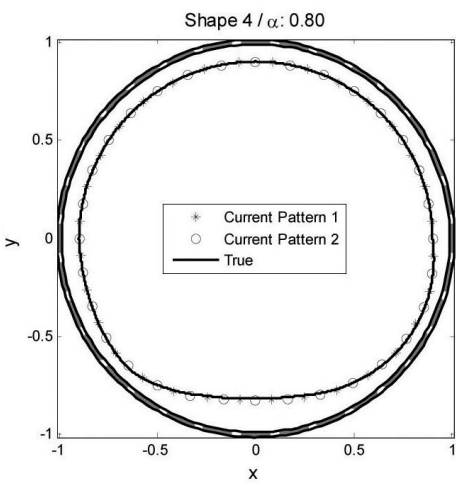

(d) Shape 4

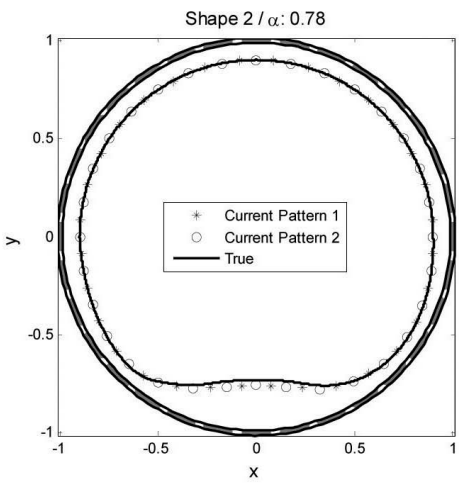

(b) Shape 2

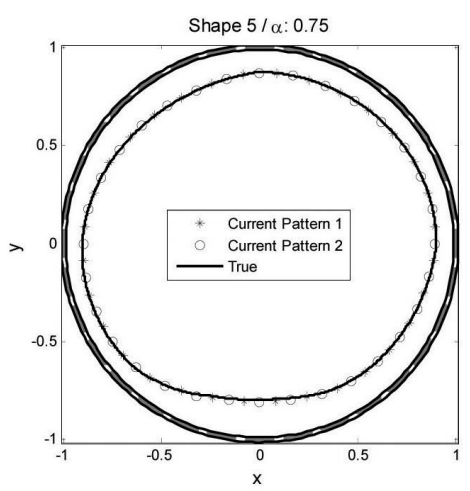

(c) Shape 5

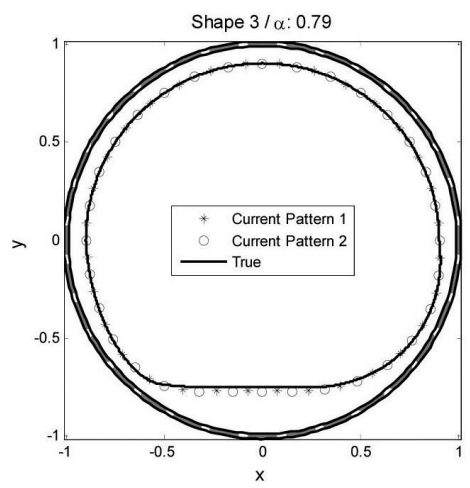

(c) Shape 3

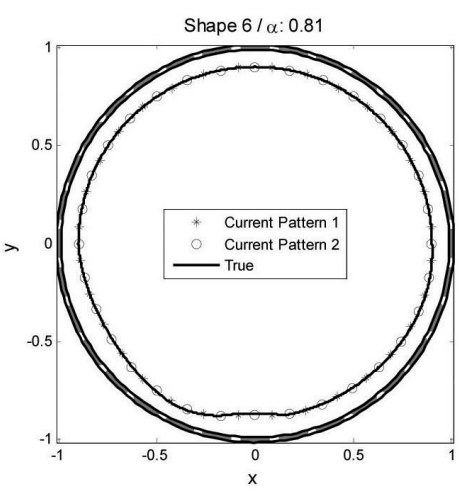

(d) Shape 6

Fig. 7. Estimated Interface for Case 3. 


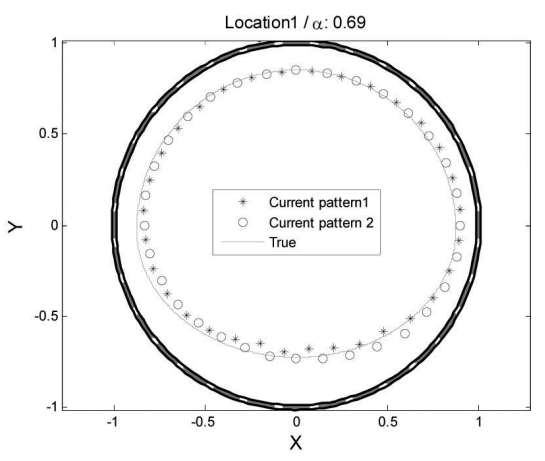

(a) Location 1

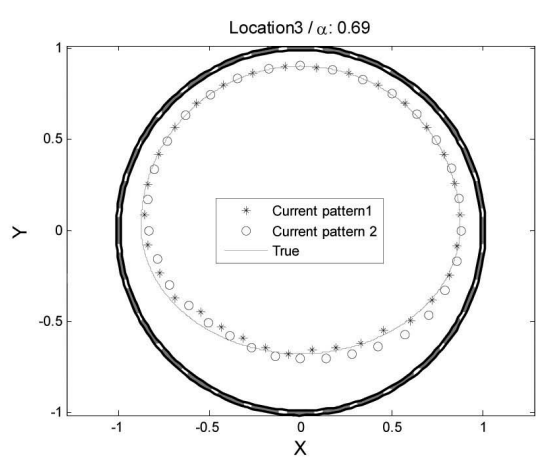

(c) Location 3

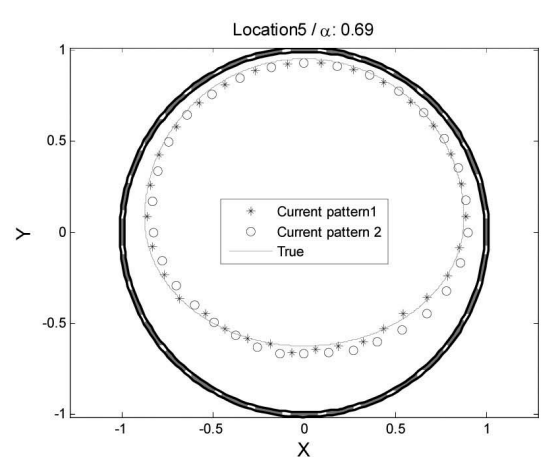

(e) Location 5

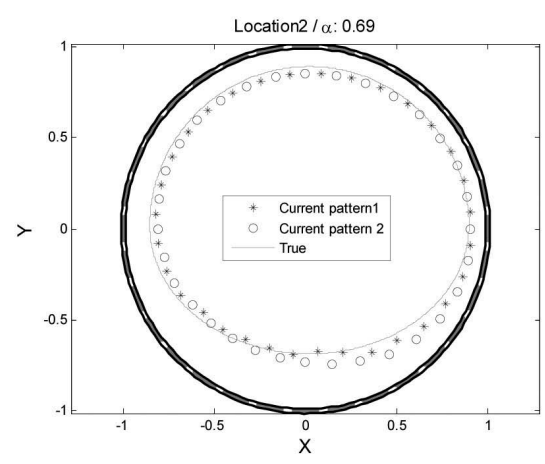

(g) Location 7

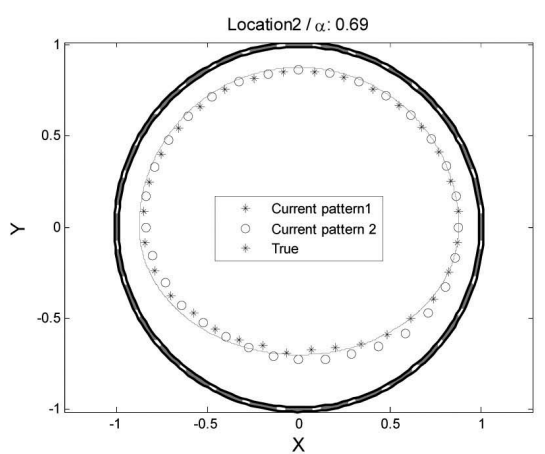

(b) Location 2

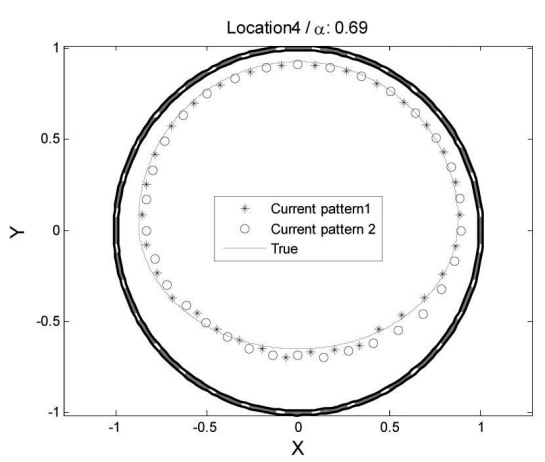

(d) Location 4

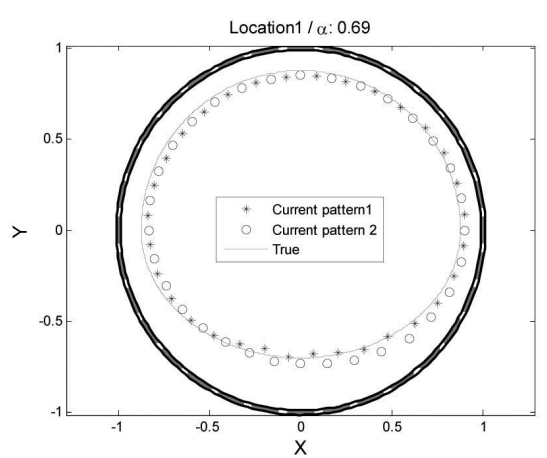

(f) Location 6

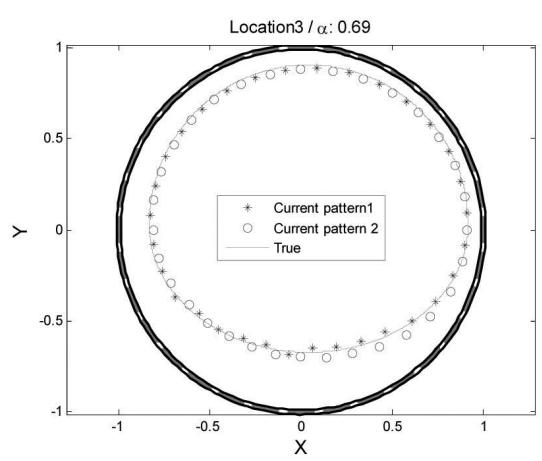

(h) Location 8

Fig. 9. Estimated Interface for Static Phantom Experiments. 


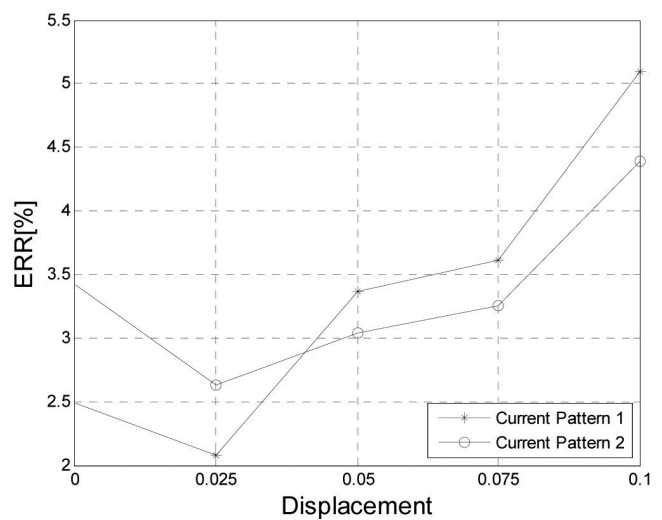

(a) Locations 1-5

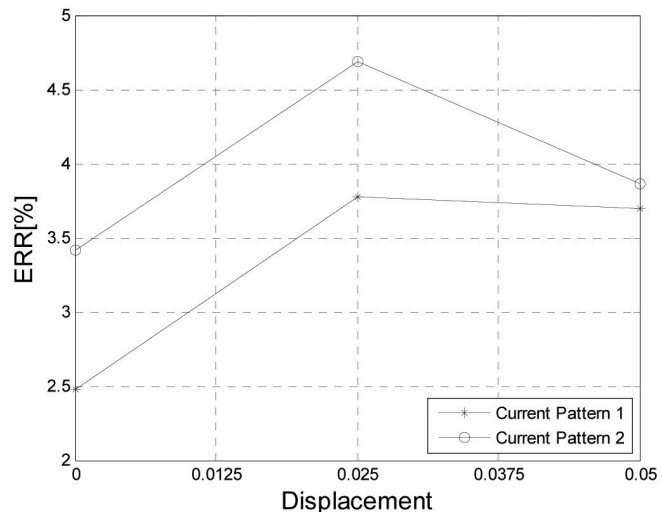

(b) Locations 6-8

Fig. 10. Estimation Error for Static Phantom Experiments.

experiments were done as the gas core was moving toward the electrode and the remaining three were done for moving gas cores along a 45 degree line toward the gap. The estimation errors are reported in Fig. 10 and the maximum was less than $5.5 \%$, which is larger than in the numerical experiments. This may have been due to the signal noise from the electric devices and the non-uniform electrode conditions mentioned above.

\section{CONCLUSIONS}

In the present work, a conductance method was employed to estimate the liquid film thickness in an annular flow through a circular pipe. The voltage difference between two adjacent electrodes was converted into the liquid film thickness based on a look-up table prepared for the concentric annular flows. Extensive numerical and static phantom experiments with 32 electrodes were carried out and the results show that the liquid film thickness can be estimated within a $5.5 \%$ error. In this work, the verification of the proposed technique was limited to static phantom experiments. The future work will be an extension of the technique to actual liquid flow.

\section{ACKNOWLEDGMENTS}

This work was supported by the research grant from the Chungbong Academic Research Fund of the Jeju National University in 2010.

\section{REFERENCES}

[1] S. Saitoh, H. Daiguji and H. Hihara H, "Correlation for Boiling Heat Transfer of R-134a in Horizontal Tubes Including Effect of Tube Diameter," Int. J. Heat Mass Transfer, 50, 5215-5225 (2007).

[2] R. Revellin and J.R. Thome, "A Theoretical Model for the Prediction of the Critical Heat Flux," Int. J. Heat Mass Transfer, 51, 1216-1225 (2008).

[3 ] C.B. Tibiriçá, F.J. do Nascimento and G. Ribatski "Film Thickness Measurement Techniques Applied to MicroScale Two-Phase Flow Systems," Exp. Therm. Fluid Sci., 34, 463-473 (2010).

[ 4 ] J.E.R. Coney, E.A.M. El-Shafei and C.G.W. Sheppard, “A Dual Laser Beam Method for Wavy Film Thickness Measurement," Optics Lasers Eng., 11, 1-14 (1989).

[5] T. Ursenbacher, L. Wojtan and J.R. Thome, "Interfacial Measurements in Stratified Types of Flow. Part I: New Optical Measurement Technique and Dry Angle Measurements," Int. J. Multiphase Flow, 30, 107-124 (2004).

[6] S.C.M. Yu, C.P. Tso and R. Liew, "Analysis of Thin Film Thickness Determination in Two-phase Flow Using a Multifiber Optical Sensor," Appl. Math. Modelling, 20, 540-548 (1996).

[7] D. Schubring, A.C. Ashwood, T.A. Shedd and E.T. Hurlburt, "Planar Laser-Induced Fluorescence (PLIF) Measurements of Liquid Film Thickness in Annular Flow Part I: Methods and Data," Int. J. Multiphase Flow, 36, 815-824 (2010).

[ 8 ] Q. Lu, N.V. Suryanarayana and C. Christodoulu, "Film Thickness Measurement with an Ultrasonic Transducer," Exp. Thermal Fluid Sci., 7, 354-361 (1993).

[9] P.C. Pedersen, Z. Cakareski and J.C. Hermanson, "Ultrasonic Monitoring of Film Condensation for Applications in Reduced Gravity," Ultrasonics, 38, 486-490 (2000).

[10] P. Stahl and P.R. Rohr, "On the Accuracy of Void Fraction Measurements by Single-Beam Gamma-Densitometry for Gas-Liquid Two-Phase Flows in Pipes," Exp. Thermal Fluid Sci., 28, 533-544 (2004).

[11] Y. Saito, K. Mishima, Y. Tobita, T. Suzuki and M. Matsubayashi, "Measurements of Liquid-Metal TwoPhase Flow by Using Neutron Radiography and Electrical Conductivity Probe," Exp. Thermal Fluid Sci., 29, 323-330 (2005).

[12] M.W.E. Coney, "The Theory and Application of Conductance Probes for the Measurement of Liquid Film Thickness in Two-Phase Flow," J. Phys. E: Scientific Instrum., 6, 903-910 (1973).

[13] T. Fukano, "Measurement of Time Varying Thickness of Liquid Film Flowing with High Speed Gas Flow by a 
Constant Electric Current Method," Nucl. Eng. Des., 184, 363-377 (1998).

[14] G. Conte, "Film Thickness Variation about a T-Junction," Int. J. Multiphase Flow, 29, 305-328 (2003).

[15] G. Geraci, B.J. Azzopardi, and H.R.E. van Maanen, "Effect of Inclination on Circumferential Film Thickness Variation in Annular Gas/Liquid Flow," Chem. Eng. Sci. 62, 3032-
3042 (2007).

[16] S. Liu, J. Li and Q. Chen, "Visualization of Flow Pattern in Thermosyphon by ECT," Flow Meas. Instrum., 18, 216-222 (2007).

[17] D.S. Holder, Electrical Impedance Tomography: Method, History and Applications, IOP Publishing Ltd, Bristol and Philadelphia (2005). 